\title{
Interactions between human neutrophils and mucin-coated surfaces
}

\author{
Tomas Sandberg $\cdot$ Jan Carlsson $\cdot$ Marjam Karlsson Ott
}

Received: 17 May 2008/Accepted: 10 September 2008

(C) Springer Science+Business Media, LLC 2008

\begin{abstract}
Recently, we showed microscopically that bovine (BSM), porcine (PGM) and human (MG1) mucin coatings could suppress the adhesion of neutrophils to a polyethylene terephthalate-based model biomaterial (Thermanox). Here, using the release of reactive oxygen species (ROS) as a marker of material-induced neutrophil activation, the strong surface-passivating effects of these mucin coatings were corroborated. Under optimal adsorption conditions, all mucin species performed equally well, thus indicating a high degree of functional homology between the mucins. Cell adhesion and morphology correlated well with the release of ROS. Quartz crystal microbalance (QCM-D) analysis linked low neutrophil activation to efficient mucin surface-shielding. Interestingly, the shielding power appeared equal for thick expanded and thin compact mucin coatings. Combined mucin-serum coatings were found to be highly surface-passivating. Particularly, since our data suggested partly synergistic mucin-serum action, we highlight the possibility that pre-adsorbed mucins could provide favorable support for adsorbing host components.
\end{abstract}

\section{Introduction}

Successful biomaterial-host integration concerns the control of several natural defense mechanisms intended to act in response to foreign matter [1]. Over the years, many different strategies have been developed to lower the adverse effects caused by implanted foreign materials. A widely used concept has been to coat the implant with a

T. Sandberg $(\bowtie) \cdot J$. Carlsson $\cdot$ M. Karlsson Ott Department of Physical and Analytical Chemistry, Division of Surface Biotechnology, BMC, Uppsala University, 75123 Uppsala, Sweden

e-mail: tomas.sandberg@ytbioteknik.uu.se biocompatible substance that can facilitate favorable host reactions [2]. Working along the same line, our research focuses on biomacromolecules collectively known as mucins, a diverse class of highly charged and highly glycosylated proteins found at the mucosal interfaces which separates "self" from "non-self" in all vertebrates [3]. The mucins are large ( $\geq 1 \mathrm{MDa})$, complex, amphiphilic molecules with naked globular "protein domains" interspersed between diversely glycosylated linear "mucin domains" [3-5]. They exist both in mucosal secretions as well as bound to the epithelial cell membranes [3] and play important roles in various interfacial in vivo processes, e.g. mastication, deglutination, speaking, eye blinking, gut protection, tumor metastasis and bacterial colonization [5, 6]. Particularly, the numerous and diverse glycosylation, polyanionic character, and large molecular dimension of the mucins contribute to their biological function as mediators and protectors of the epithelial surface $[4,6]$.

In line with their protective role in vivo, our group in the past showed that surface coatings with a mucin fraction from bovine saliva (BSM) are capable of reducing the uptakes of proteins, cells and bacteria to various polymeric substrates [7, 8]. Lately, we have gained an increased interest in the compositional aspects governing the performance of the BSM coating. In addition, mucins from porcine (PGM) and human (MG1) sources have been included in the research platform in an attempt to address regulatory issues related to the medical use of substances derived from bovine tissue materials. As a result, we have developed a panel of well-defined mucins of different structural and compositional status intended for biocompatibility studies on mucin coatings [9].

The innate immune system constitutes an early-phase, non-specific barrier against external threats, normally consisting of exogenous cells, bacteria and viruses [10]. Here, 
we focus on the neutrophils, which are cellular members of the innate immune system and important mediators of biomaterial-induced inflammation [11]. Earlier studies have pointed to important in vivo relationships between mucins and neutrophils; e.g. the human salivary MUC7 mucin has been found in association with neutrophils in the oral cavity [12] and the neutrophil release product elastase has been shown to increase epithelial mucin secretion [13]. Of more direct relevance to the application of mucins as biomaterial coatings, a previous study suggested the commercial mucin preparation from which we purified the present more albumin- and aggregate-free BSM, to inhibit lymphocyte surface function through steric hindrance [14]. Another study reported that ocular mucins from dry eye patients, which are underglycosylated and adopt compact surface conformations, activate neutrophils to a lower degree than their normal, more diffusely bound counterparts [15].

Recently, we performed a microscopic evaluation of the interactions between human neutrophils and substrates of a polyethylene terephthalate-based model biomaterial (Thermanox) coated with the BSM, PGM and MG1 mucins, respectively [16]. Our results showed that all mucins could be used to effectively suppress the adhesion of neutrophils to the material surface. Furthermore, in good agreement with previous findings [14, 15], it was indicated that dense mucin surface packing is a prerequisite for good coating performance.

The present study is a more in-depth evaluation of the interactions between neutrophils and mucin-coated substrates. Using the same model material (Thermanox), differently mucin-coated substrates were incubated with neutrophils under physiological buffer- and temperature conditions. The neutrophil activation was thereafter studied, in terms of the production of reactive oxygen species (ROS), and for selected coatings, in terms of the cell adhesion and morphology. Correlations were made between the neutrophil activation and the individual mucin uptakes on the substrates. In complement to previous studies on mucin-leukocyte interaction $[14,15]$, we employed the quartz crystal microbalance technique supplemented with dissipation monitoring (QCM-D) to evaluate possible differences in organization between different mucin coatings. Finally, to predict the behavior of mucin-coated materials in vivo, the neutrophil response towards mucin-coated substrates contacting human serum was studied.

\section{Materials and methods}

\subsection{General chemicals, proteins and buffers}

For general protein dilutions and coating washing steps, a $20 \mathrm{mM}$ sodium/potassium phosphate buffer of $\mathrm{pH} 7.4$, with a total ionic strength of $150 \mathrm{mM}$ (adjusted with $\mathrm{NaCl}$ ) and a $\mathrm{Na} / \mathrm{K}$ ratio of 33 (PBS20), was used. High salt condition was generated using PBS20 with a $\mathrm{NaCl}$-adjusted total ionic strength (PBS20-1 M). For low pH conditions $20 \mathrm{mM}$ sodium acetate of $\mathrm{pH} 4.0$ with a total ionic strength of $150 \mathrm{mM}$ (ABS20) was used. Alternatively, PBS20 was mixed in situ with $1 \mathrm{M} \mathrm{HCl}$ in a 10:1 ratio giving $\mathrm{pH} 1$ (PBS20-pH1). The buffers were prepared by mixing the respective acid-base pairs in appropriate proportions. All cell experiments were performed in Hank's balanced salt solution (HBSS; Sigma H8264). Human serum albumin (HSA) was purchased from Sigma (A3782). Human serum, originating from seven apparently healthy individuals, was prepared as known in the art, stored frozen in aliquots at $-80^{\circ} \mathrm{C}$ and thawed at $37^{\circ} \mathrm{C}$ immediately before use.

Chemicals and solvents were of highest purity available and obtained from commercial sources unless otherwise stated.

\subsection{Preparation of bovine, porcine and human mucins}

Mucins of bovine salivary (BSM), porcine gastric (PGM) and human salivary (MG1) origins were prepared according to recently established protocols [9]. Briefly, partially purified bovine submaxillary gland mucin (Sigma M3895), partially purified pig gastric mucin (Sigma M1778) and human whole saliva were dissolved, equilibrated and then gel-filtered, before being desalted and finally freeze-dried. For the bovine material an additional fractionation step involving anion exchange chromatography was used. All mucin preparations were stored desiccated until use.

While the BSM and PGM mucins were relatively pure the MG1 mucin contained significant amounts of bound non-mucin material, including albumin, which constituted $1.6 \mathrm{wt} . \%$ of the MG1 preparation. In addition, the MG1 mucin contrasted the other, mainly random-coiled mucins by adopting compact solution conformation. The mucin fractions, whose predominant species had molar masses in the range $0.80-4.2 \mathrm{MDa}$, were equilibrated in their respective buffers for at least $12 \mathrm{~h}$ before any experiment. Occasionally, comparisons were made between high- and low-molar mass forms of the BSM and PGM mucins, then denoted by indices I and II, respectively. Unless noted, BSM and PGM refer to low-molar mass mucin fractions.

\subsection{Model substrate and coating procedures}

All coatings were formulated on a polymeric model substrate, Thermanox (Nunc). The used, treated surface of this substrate is generally referred to as "surface-oxidized polyethylene terephthalate (PET)" and is a relevant model for many soft implant surfaces [17]. Water contact angle analysis showed the treated surface to be dynamic, with an 
initial contact angle changing from $75^{\circ}$ to $55^{\circ}$ upon $1 \mathrm{~h}$ in water. Using the treated surface towards the bulk medium, all coating procedures were performed at room temperature in 24-well cell culture plates (Costar 3524; Corning Inc.)

Thermanox substrates were spot-coated with either the MG1 $(0.125 \mathrm{mg} / \mathrm{ml})$, BSM $(0.25 \mathrm{mg} / \mathrm{ml})$ or PGM $(0.25 \mathrm{mg} / \mathrm{ml})$ mucins for $20 \mathrm{~h}$ at room temperature in PBS20. The formed coatings were referred to as "regular mucin coatings". The concentrations used for the formulation of these coatings were based on the near-plateau concentrations recently found for a hydrophobic model surface [9]. In addition, the low-molar mass forms of BSM and PGM (indexed II) were adsorbed at high mucin concentration $(1 \mathrm{mg} / \mathrm{ml})$, at high ionic strength $(1 \mathrm{M})$ and at low $\mathrm{pH}(\mathrm{pH} 1)$. The BSM-II mucin was also adsorbed at $\mathrm{pH}$ 4. The latter four types of coatings were referred to as "non-regular mucin coatings". In another experimental setup, regular coatings of BSM-II and PGM-II, formulated as above $(0.25 \mathrm{mg} / \mathrm{ml}$ in PBS20), were incubated for $4 \mathrm{~h}$ with 1 vol.\% human serum diluted in PBS20 ("composite mucin coatings"). Table 1 summarizes the different coating formulation conditions used. All surfaces were washed three times with PBS20 after each adsorption step to remove loosely bound material. Finally, all prepared surfaces were stored overnight in PBS20 until used.

\subsection{Surface protein concentrations}

The surface concentration of respective single component coating (mucin or human serum proteins) was quantified using the Micro BCA (mBCA) assay [18]. In brief, following the incubation of buffer-washed samples with the mBCA reagent (Pierce 23232) for $4 \mathrm{~h}$ at $37^{\circ} \mathrm{C}$ and $48 \mathrm{~h}$ at $8^{\circ} \mathrm{C}$, the relative amounts of adsorbed material were quantified spectrophotometrically (Multiskan MS) at $570 \mathrm{~nm}$. Standards, prepared from solutions of known analyte concentrations, were assayed in parallel and the surface concentrations of protein were calculated using constructed standard curves in combination with known substrate surface areas. For the human serum standard pool (see above), whole serum was assumed to have a total protein concentration of $70 \mathrm{mg} / \mathrm{ml}$ [19].

The mBCA assay was combined with mass-calibrated (ellipsometry) enzyme-linked lectin (ELLA) and immunosorbent (ELISA) assays to determine the individual amounts of mucin and human serum proteins, respectively, in the composite layers. For a detailed description of the used analytical procedure, see [20]. Briefly, mucin and human serum proteins were targeted using jacalin and antihuman whole serum IgG, respectively. The amount of surface-bound analyte was then quantified using enzymelinked probes specific for jacalin and $\mathrm{IgG}$, respectively. Finally, the amount of each coating component was quantified from combining the ELLA/ELISA data with data from the mBCA analysis. As demonstrated in [20], desorption of mucin upon addition of serum proteins was low.

\subsection{Neutrophil preparation}

Granulocytes, of which the neutrophils constitute the predominant fraction, were isolated from heparinized peripheral blood on the day of the experiment from apparently healthy individuals essentially following the method of Håkansson and Venge [21]. Neutrophil counts were in the range $28-45 \times 10^{9}$ cells/ 1 with a cell

Table 1 Compilation of coating formulation conditions

\begin{tabular}{|c|c|}
\hline Coating & Formulation conditions \\
\hline \multicolumn{2}{|c|}{ Regular mucin coatings } \\
\hline Mucin & Mucin adsorbed at 0.125 (MG1) or 0.25 (BSM-I, BSM-II, PGM-I and PGM-II) mg/ml for approximately $20 \mathrm{~h}$ \\
\hline \multicolumn{2}{|c|}{ Non-regular mucin coatings } \\
\hline $\begin{array}{l}\text { Mucin, Hi conc. } \\
\text { Mucin, } \mathrm{pH} 1\end{array}$ & $\begin{array}{l}\text { Regular mucin coating although adsorbed at high concentration }(1.0 \mathrm{mg} / \mathrm{ml}) \text {, at } \mathrm{pH} 1 \text { (PBS20-pH1), at } \mathrm{pH} 4(\mathrm{ABS} 20) \\
\text { or at } 1 \mathrm{M} \text { ionic strength (PBS20-1 M) }\end{array}$ \\
\hline \multicolumn{2}{|l|}{ Mucin, $\mathrm{pH} 4$} \\
\hline \multicolumn{2}{|l|}{ Mucin, $1 \mathrm{M}$ salt } \\
\hline \multicolumn{2}{|c|}{ Composite mucin coatings } \\
\hline Mucin + serum $1 \%$ & Regular mucin coating incubated with 1 vol.\% human serum for $4 \mathrm{~h}$ \\
\hline \multicolumn{2}{|l|}{ Controls } \\
\hline Serum $1 \%$ & Human serum adsorbed at 1 vol. $\%$ for $4 \mathrm{~h}$ \\
\hline PBS20 & PBS20 buffer incubated for approximately $20 \mathrm{~h}$ \\
\hline
\end{tabular}

All coatings were formulated on Thermanox substrates at room temperature. If not otherwise indicated, PBS20 buffer of pH 7.4 was used. The substrates were rinsed with PBS20 after each coating step and stored in the same buffer until used. Indices I and II denote high- and low-molar mass mucin variants, respectively 
contamination of less than $10 \%$. Before use, cell suspensions were diluted in room-tempered HBSS to 650,000 cells $/ \mathrm{ml}$. Neutrophils were used within $2-3 \mathrm{~h}$ after preparation.

\subsection{Quantification of reactive oxygen species (ROS)}

The total neutrophil production of reactive oxygen species (ROS) following contact with the differently coated Thermanox substrates was quantified using luminolamplified chemiluminescence [22].

Coated substrates were rinsed twice with HBSS and transferred, keeping the coated side towards the bulk medium, to 24-well optiplates intended for chemiluminescent measurements (Perkin-Elmer). Neutrophils, added immediately in $0.5 \mathrm{ml}$ aliquots to the rinsed substrates (325,000 cells/sample), were followed by $100 \mu \mathrm{l}$ of luminol-horseradish peroxidase reagent $(50 \mu \mathrm{M}$ luminol and $4 \mathrm{U} / \mathrm{ml}$ horseradish peroxidase; purchased from Sigma) [22]. The real-time production of ROS was monitored using a Victor $^{2} 1420$ luminometer (Wallac). Plates were incubated at $37^{\circ} \mathrm{C}$ between all measurements. The delay time between cell addition and the first reading was approximately $4 \mathrm{~min}$. After completed measurements, cell suspensions were removed and the neutrophil-exposed surfaces fixed for the microscopic analyses using 1.5\% glutaraldehyde (Merck).

\subsection{Microscopic evaluation}

The neutrophil adhesion and morphology were analyzed using a LEO Gemini 1530 scanning electron microscope (SEM). Specifically, fixed samples were rinsed quickly with MilliQ-grade water, dehydrated stepwise using water solutions of ethanol (50-100 vol.\%) and finally supercritically dried in liquid $\mathrm{CO}_{2}$. After subsequent gold sputtering, samples were imaged using an acceleration voltage of $2 \mathrm{kV}$. Alternatively, the fixed samples were stained for 30 min with 5\% Giemsa stain (Sigma GS-500), rinsed quickly with MilliQ-grade water and dried before evaluation by means of light microscopy (Carl Zeiss).

Relative mean cell counts (C.C.) and mean cell areas (C.A.) compared to the non-coated substrate control were analyzed using the ImageJ software (NIH; version 1.36b). Cells were counted per one microscopic view, which correlated to approximately $0.02 \mathrm{~mm}^{2}$ for the SEM micrographs and $0.3 \mathrm{~mm}^{2}$ for the light microscopy images.

2.8 Quartz crystal microbalance with dissipation monitoring (QCM-D)

Structural information about selected mucin coatings was gained using the QCM-D technique (Q-Sense D300 system, Q-Sense AB). This technique allows for realtime studies of the molecular adsorption to different materials. Here, we used a commercial polystyrenecoated QCM crystal (Q-Sense $\mathrm{AB}$ ) as a model for a hydrophobic polymeric material. Before use, the sensor crystal and all tubings were cleaned with $0.1 \mathrm{M} \mathrm{NaOH}$ in $10 \%$ ethanol for $30 \mathrm{~min}$ and thereafter thoroughly rinsed with MilliQ-grade water. The measuring chamber was cleaned in sequence using 2\% Hellmanex (Hellma $\mathrm{GmbH}$ ) and MilliQ-grade water, respectively. Freshly cleaned sensor crystals were installed into the measuring chamber and equilibrated at $22 \pm 0.01^{\circ} \mathrm{C}$ with degassed PBS20 buffer until a stable baseline was reached. Following this, $0.5 \mathrm{ml}$ of pre-tempered $\left(22^{\circ} \mathrm{C}\right)$ protein solution was injected into the chamber and allowed to adsorb until steady-state conditions were reached. Finally, the sensor surface was rinsed 3-4 times with PBS20 in order to remove loosely bound material. The changes in resonance frequency $(\Delta f$; related to attached mass) and energy dissipation ( $\Delta D$; related to frictional losses in the adlayer) were monitored continuously during adsorption and plots of $\Delta D$ against $\Delta f$ were constructed to obtain structural information regarding the formed adlayer [23]. All presented $\Delta D-\Delta f$ plots are based on the normalized fifth overtone frequency $\left(f_{5} / 5=25 / 5 \mathrm{MHz}\right)$.

The viscoelastic model of Voinova et al. [24] was used to evaluate (Q-Tools, Q-Sense $\mathrm{AB}$ ) the effective hydrodynamic thickness $\left(d_{f}\right)$, density $\left(\rho_{f}\right)$, shear viscosity $\left(\eta_{f}\right)$, and shear elastic modulus $\left(\mu_{f}\right)$ of the adsorbed films. Values for the bulk fluid density and viscosity were $1,000 \mathrm{~kg} / \mathrm{m}^{3}$ and $1 \mathrm{mPas}$, respectively, and the layer density was allowed to adopt values between those of the bulk fluid and a theoretical close-packed protein layer $\left(1,400 \mathrm{~kg} / \mathrm{m}^{3}\right)$ [25]. The characteristic relaxation time $\left(\tau_{f}\right)$ of each layer was calculated as the ratio between the shear viscosity and the shear elastic modulus [26]. Coating water contents were roughly estimated from comparing "wet" (QCM-D) and "dry" (estimated as the protein surface concentration on a Thermanox substrate coated under the same conditions) adlayer masses.

\subsection{Statistical analysis}

Generally, the data were based on 3 or more individual samples $(n \geq 3)$ and presented as the arithmetic mean \pm 1 SD. For the ROS data, analyses were performed in duplicate to quadruplicate experiments with neutrophils from 2 to 4 donors $(2 n-4 n)$. Levels of difference in significance were tested with the two-tailed non-paired Student's $t$-test. Significance levels of $* P<0.05$ and NS $=$ not significant, were used; data not marked by any of the two levels in the figures are the $t$-test controls. 


\section{Results}

\subsection{Neutrophil activation: ROS release}

Figure 1 presents the neutrophil production of reactive oxygen species (ROS) following contact with mucincoated Thermanox substrates formulated under regular conditions $(0.125$ or $0.25 \mathrm{mg} / \mathrm{ml}$ in PBS20 $\mathrm{pH}$ 7.4) according to Table 1 . Including the measurement delay time, the ROS production peaked after approximately 15 min of neutrophil exposure for all samples. All mucincoated substrates were associated with significantly lower neutrophil activation (at the 95\% confidence level) than the non-coated control (PBS20). Specifically, the substrate coated with the human mucin (MG1) showed the lowest neutrophil activation, followed by the substrate coated with the high-molar mass bovine mucin (BSM-I). Substrates coated with either of the porcine mucins (PGM-I and PGM-II) or the low-molar mass bovine mucin (BSM-II) showed only weak reductions in ROS compared to the control.

From studying the native mucosa and the high-performing and presumed thick MG1 coating, we foresaw advantages in producing thick mucin "hydrogels", which could act as physical barriers to the underlying surface. We therefore formulated the low-molar mass forms of BSM and PGM (occasionally denoted without index II) at higher mucin concentration, at higher ionic strength

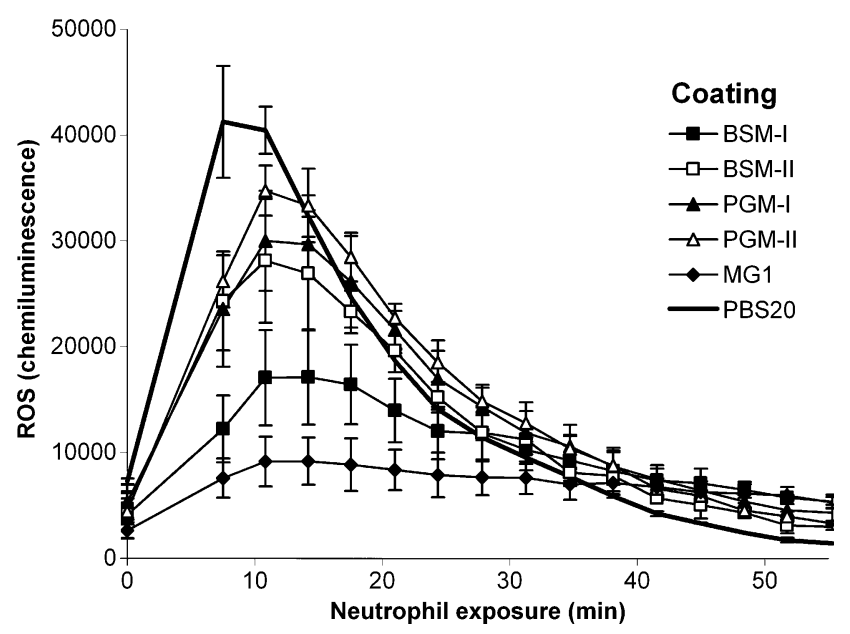

Fig. 1 Diagram showing the typical production of reactive oxygen species (ROS; expressed as luminol-amplified chemiluminescence) as a function of neutrophil exposure time for Thermanox substrates coated with bovine (BSM), porcine (PGM) and human (MG1) mucins, respectively. The mucins were adsorbed overnight at $0.125 \mathrm{mg} / \mathrm{ml}$ (MG1) and $0.25 \mathrm{mg} / \mathrm{ml}$ (BSM and PGM), respectively. Detailed coating procedures are found in Table 1. Indices I and II refer to high- and low-molar mass mucin variants, respectively, and PBS20 is the non-coated material. Error bars represent mean \pm 1 SD. $(1 n)$ and at lower $\mathrm{pH}$, conditions known to promote mucin gelation and increase surface adhesiveness [27-30]. Figure 2 summarizes the neutrophil ROS production induced by substrates coated with mucin under these conditions (except for the PGM coating formulated at low $\mathrm{pH}$ ), together with the results from Fig. 1. The data is presented as the integrated total amount of produced ROS compared to the non-coated control. As seen in the figure, the substrates coated at high mucin concentration $(1 \mathrm{mg} / \mathrm{ml})$ induced significantly lower neutrophil activation than the substrates coated under regular conditions. The same effect was observed for the BSM-coated substrate formulated under highly acidic conditions $(\mathrm{pH} 1)$. However, the substrates formulated with mucins at $\mathrm{pH} 4$ and at high ionic strength $(1 \mathrm{M})$ did not show any difference in ROS generation compared to those bearing coatings formulated under regular conditions.

Since biomaterials commonly encounter blood during implantation, we studied the effect of exposing mucincoated substrates to 1 vol.\% human serum before neutrophil contact. Figure 3 shows that the effect of serum contact on the neutrophil activation was largely reduced upon pre-coating the substrate with mucin (51 and 66\% reduced ROS production compared to the serum-coated control for the BSM- and PGM pre-coated substrates, respectively). In addition, the mucin and serum components demonstrated a combined effect, as the substrates bearing the composite coatings induced significantly lower neutrophil activation than those coated with each constituent separately.

\subsection{Neutrophil activation: cell adhesion and morphology}

The neutrophil adhesion and morphology following substrate contact were recently evaluated microscopically on substrates coated with mucins under regular conditions and at higher mucin concentration [16]. Here, we performed complementary microscopic analyses on substrates coated with BSM and PGM, respectively, at $\mathrm{pH} 1$, at $\mathrm{pH} 4$ and at $1 \mathrm{M}$ ionic strength. We found the microscopic data to correlate well with the observed trends in ROS production. That is, while the substrates coated with mucin at $\mathrm{pH} 4$ and at $1 \mathrm{M}$ ionic strength showed no improved bioperformance compared to the non-coated control (data not shown), much fewer and less spread cells were found on the substrates coated at pH 1 (Fig. 4).

As depicted in Fig. 5, very few neutrophils populated the substrates bearing mucin-serum composite coatings. These cells adopted a non-activated morphology (round without signs of extracellular processes). This correlated well with the low generation of ROS observed for these substrates. 


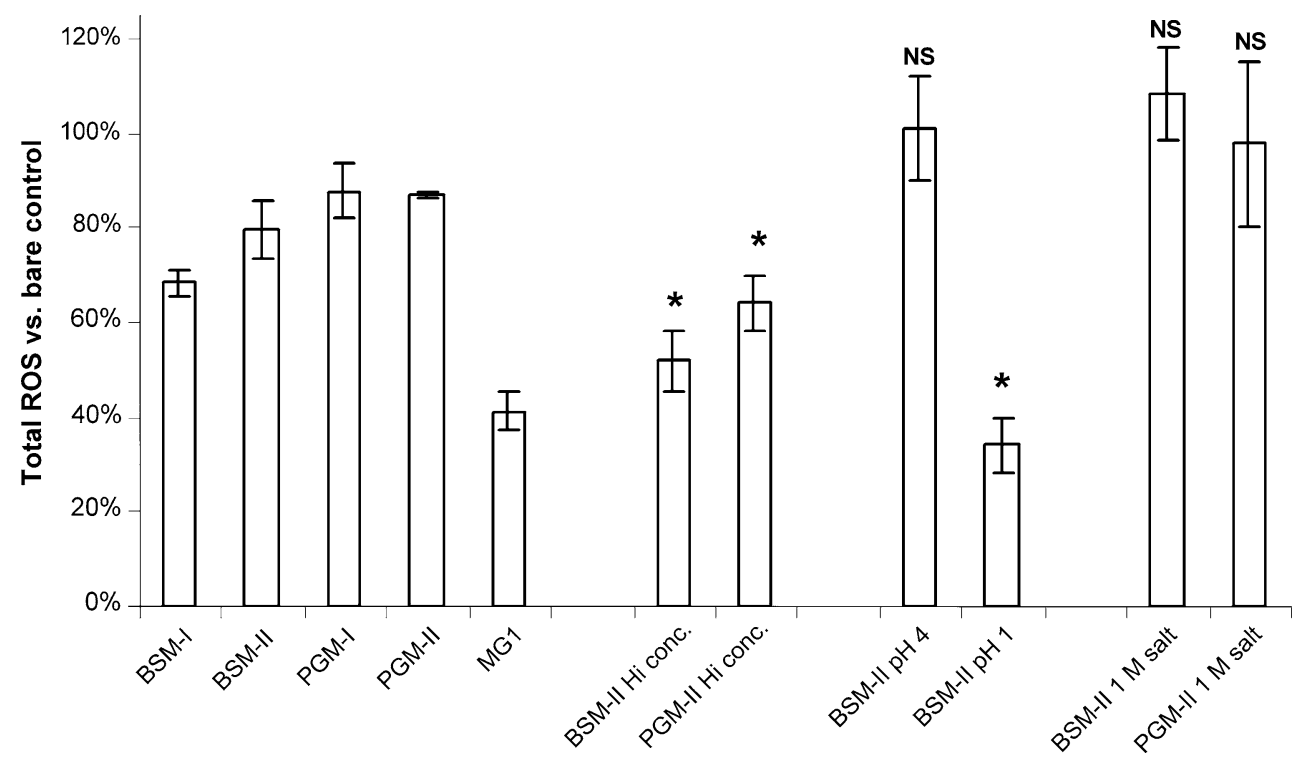

Fig. 2 Diagram summarizing the produced total amount of ROS compared to the non-coated control after $55 \mathrm{~min}$ of neutrophil exposure to differently mucin-coated Thermanox substrates. The mucins (BSM, PGM and MG1) were adsorbed overnight either at regular conditions $(0.125$ or $0.25 \mathrm{mg} / \mathrm{ml}$ in PBS20 $\mathrm{pH} 7.4)$, at high

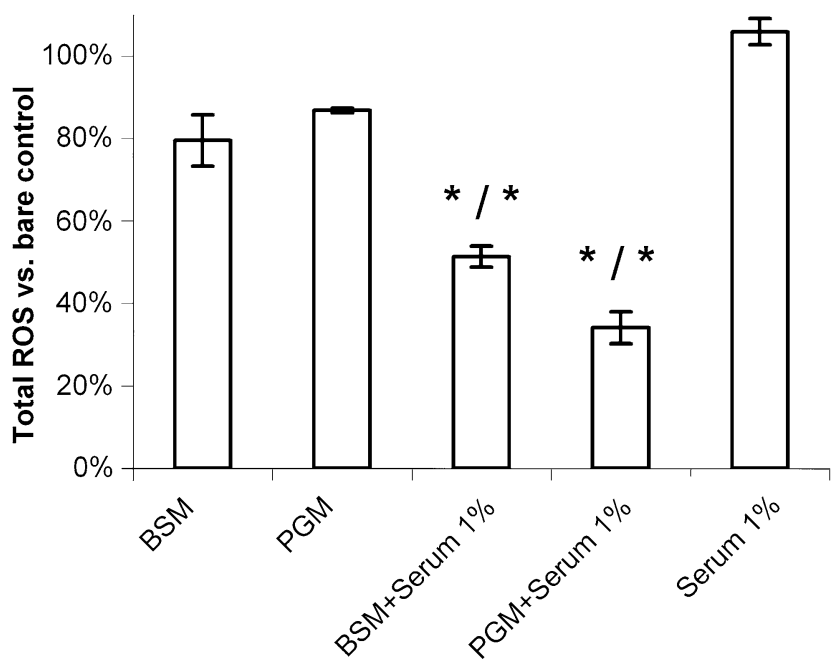

Fig. 3 Diagram summarizing the produced total amount of ROS compared to the non-coated control after $55 \mathrm{~min}$ of neutrophil exposure to Thermanox substrates coated sequentially with mucin (BSM or PGM at $0.25 \mathrm{mg} / \mathrm{ml}$ ) and human serum (1 vol.\%) according to Table 1. Error bars represent mean $\pm 1 \mathrm{SD}$ and significant differences compared to regular mucin coating (left asterisk) and serum control (right asterisk) are marked, using the following significance levels: ${ }^{*} P<0.05$ and NS $=$ not significant. $(2 n-3 n)$

\subsection{Surface protein concentrations}

In order to understand the observed differences in neutrophil activation, the amount of adsorbed protein was analyzed for all coated substrates. Figure 6 a shows that the mucin concentration $(1 \mathrm{mg} / \mathrm{ml})$, at low $\mathrm{pH}(\mathrm{pH} 1$ and $\mathrm{pH} 4)$ or at high ionic strength (1 M salt), according to Table 1. Error bars represent mean $\pm 1 \mathrm{SD}$ and levels of significance used for comparison with respective regular mucin coating are: $* P<0.05$ and $\mathrm{NS}=$ not significant. $(2 n-3 n)$

mass uptakes spanned a large interval for the mucins adsorbed under regular conditions; the lowest surface concentration was seen for the BSM-II coating $\left(2.5 \mathrm{mg} / \mathrm{m}^{2}\right)$, followed by the BSM-I and PGM-II coatings (approximately $3.9 \mathrm{mg} / \mathrm{m}^{2}$ ), and the PGM-I and MG1 coatings ( 8.3 and $11 \mathrm{mg} / \mathrm{m}^{2}$, respectively). As illustrated in Fig. $6 \mathrm{~b}$, higher mass uptakes were reached when formulating the low molar mass BSM and PGM mucin fractions (indices omitted) at higher mucin concentration, at higher ionic strength and at $\mathrm{pH} 1$. We find that only the BSM coating formulated at $\mathrm{pH} 4$ shows a similar surface concentration as its corresponding regular coating.

For the mucin-serum coatings additional analyses were performed combining the mBCA assay with mucin- and whole serum sensitive assays [20]. These analyses revealed large contents of serum proteins in the composite coatings (Fig. 7). Assuming that $100 \%$ of the pre-adsorbed mucin layer remained after serum addition [20], we could estimate the fractions of serum proteins in the formed BSMserum and PGM-serum composites as 71 and 63 wt.\% of the total adsorbed mass, respectively. These values corresponded to serum uptakes of 73 and $79 \%$ of that for the bare substrate (i.e. the serum-coated control), respectively. It follows that, the total surface protein concentration increased significantly upon serum addition to the substrates bearing regular mucin coatings. Notably however, the resulting mucin-serum composites contained similar total amounts of protein as the serum-coated control. In detail, while the PGM-serum composite showed slightly 

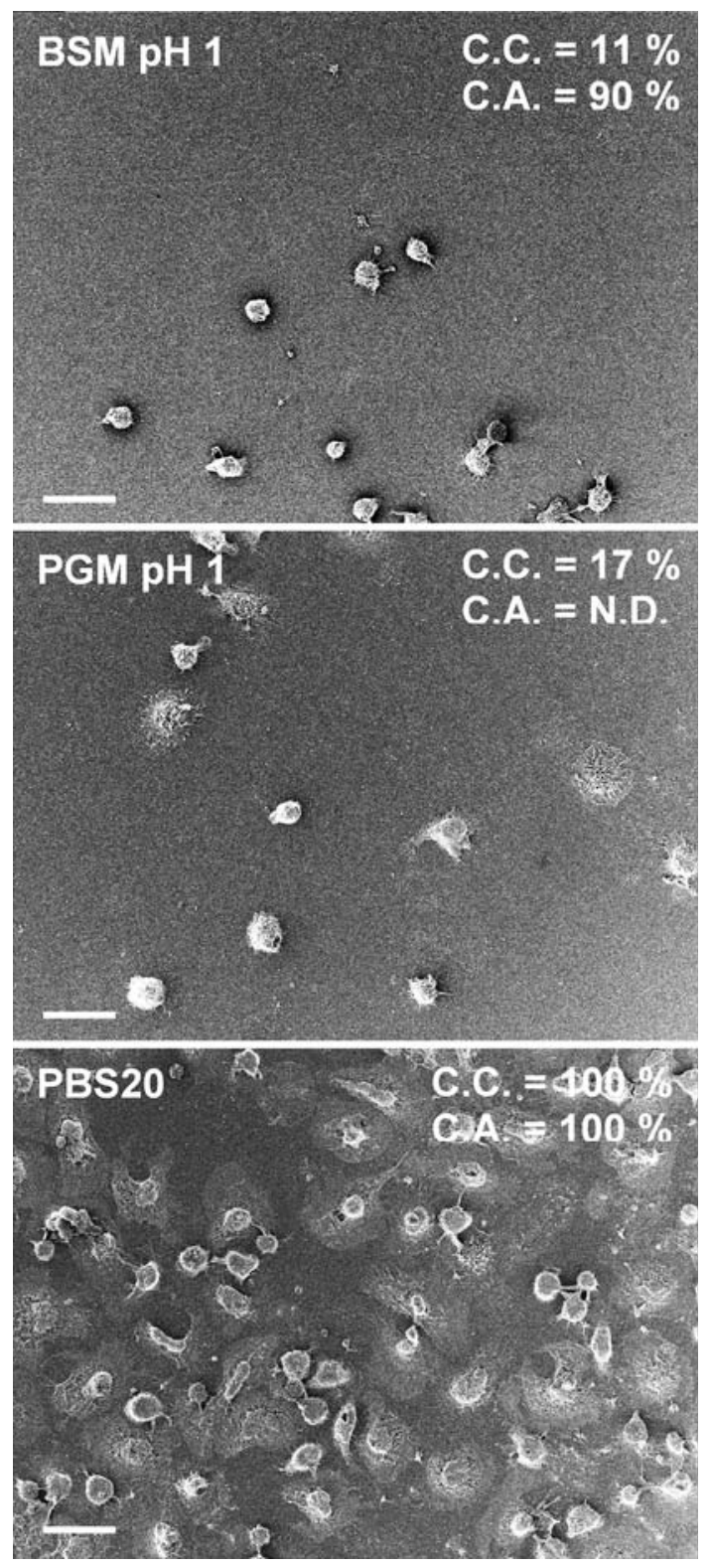

Fig. 4 SEM images showing Thermanox substrates coated with mucin (BSM or PGM) at $\mathrm{pH} 1$ after 55 min of neutrophil exposure. Non-coated control (PBS20) is shown for comparison. Detailed coating procedures are found in Table 1 . The relative numbers refer to mean cell counts (C.C.) and mean cell spreading (contact area; C.A.) compared to the non-coated control. Scale bar represent $20 \mu \mathrm{m}$. N.D. is not determined. $(3 n-4 n)$

higher total surface concentration than the serum control, no significant difference was observed for the BSM-serum composite.

To explore the effect of different mucin surface concentrations on the serum uptake, serum was added according to the above (1 vol.\%) to BSM- and PGM-coated substrates formulated at increasingly high mucin concentrations $(0.25,0.5$ and $1.0 \mathrm{mg} / \mathrm{ml})$. Not surprisingly, the uptake of serum components decreased for substrates
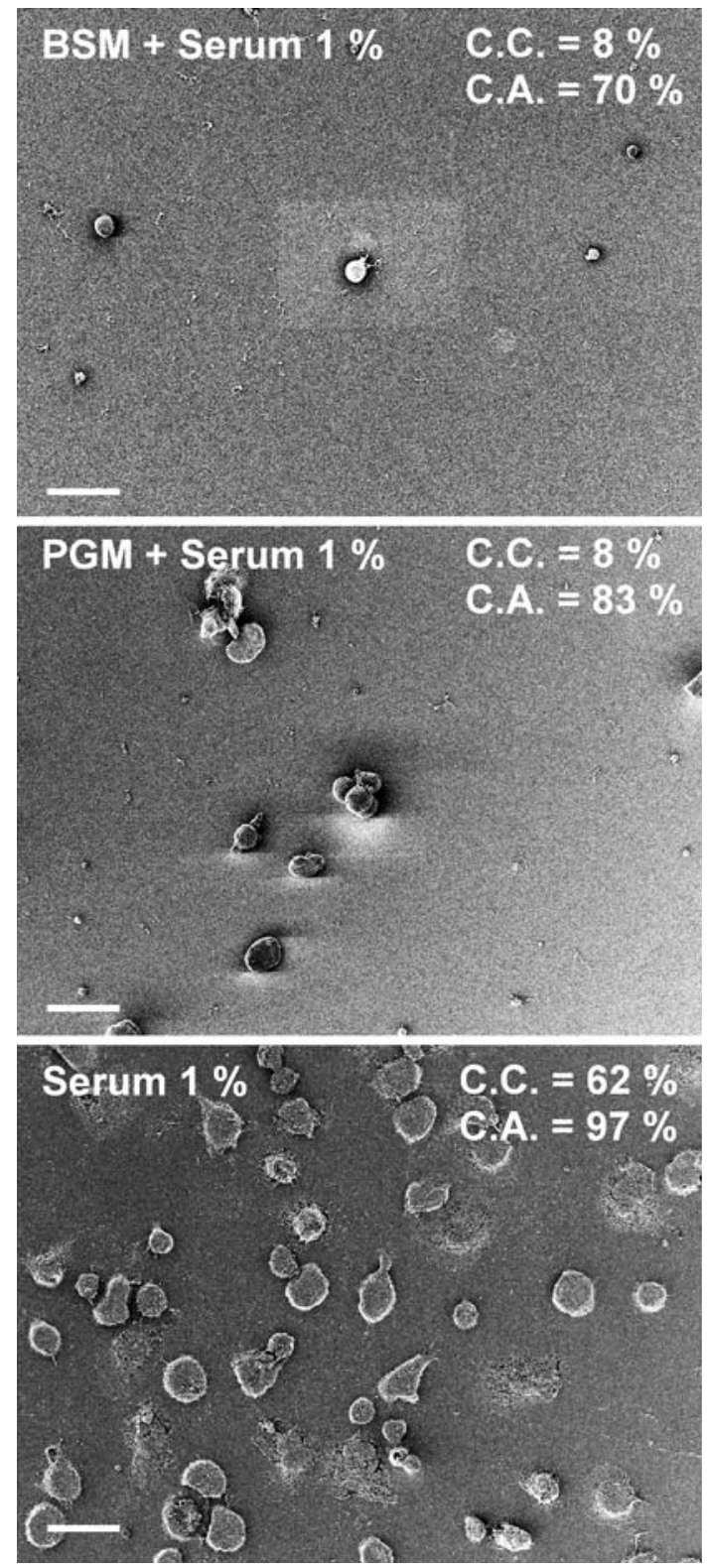

Fig. 5 SEM images showing Thermanox substrates coated sequentially with mucin (BSM or PGM) and human serum (1 vol.\%) after 55 min of neutrophil exposure. Serum-coated control is shown for comparison. Detailed coating procedures are found in Table 1. The relative numbers refer to mean cell counts (C.C.) and mean spreading (contact area; C.A.) compared to the non-coated control (PBS20). Scale bar represent $20 \mu \mathrm{m}$. $(3 n-4 n)$

coated at higher mucin concentrations (data not shown). However, the fraction of serum components in the composite coatings reached a minimum at a significant level, constituting at lowest $31 \mathrm{wt} . \%$ of the total adlayer mass.

\subsection{QCM-D evaluation of mucin coating properties}

The QCM-D technique was employed to validate potential differences in physical properties between differently 

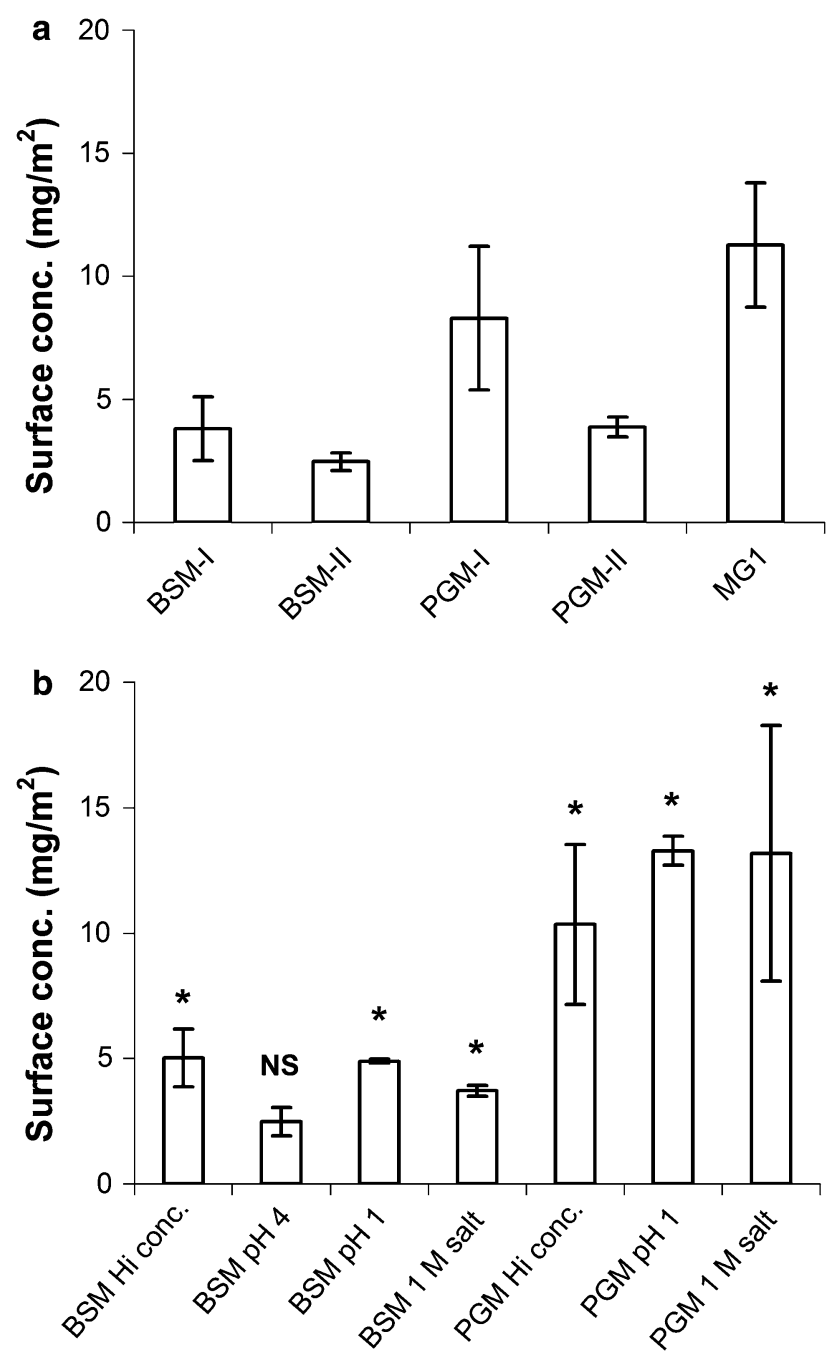

Fig. 6 Mucin surface concentrations on differently coated Thermanox substrates. Regular coatings of BSM, PGM and MG1, respectively, formulated at regular mucin concentration $(0.125$ or $0.25 \mathrm{mg} / \mathrm{ml}$ ) (a). Non-regular coatings of BSM and PGM (low molar mass variants; index II omitted for improved readability), respectively, formulated at high mucin concentration $(1.0 \mathrm{mg} / \mathrm{ml})$, low $\mathrm{pH}$ (pH 1 and $\mathrm{pH} 4$ ) and high ionic strength (1 M salt), respectively (b). Detailed coating procedures are found in Table 1. Samples were analyzed using the mBCA assay according to section 2.4. Significant differences compared to the regular mucin coatings are marked with asterisks (b), using the following significance levels: $* P<0.05$ and $\mathrm{NS}=$ not significant. Error bars represent mean $\pm 1 \mathrm{SD} .(n=3)$

formed mucin coatings. Figure 8 presents the shifts in dissipated energy $(\Delta D)$ recorded for given shifts in frequency $(\Delta f)$ for final (washed and relaxed) mucin adlayers formed on a polystyrene model surface. Generally, a $\Delta D-\Delta f$ plot eliminates time as an explicit adsorption parameter and provides insight into the viscoelastic nature of the formed adlayer. Specifically, the slope of the line constructed between the initial and final point of adsorption, $K_{D-f}(=\Delta D / \Delta f)$, scale differently with different

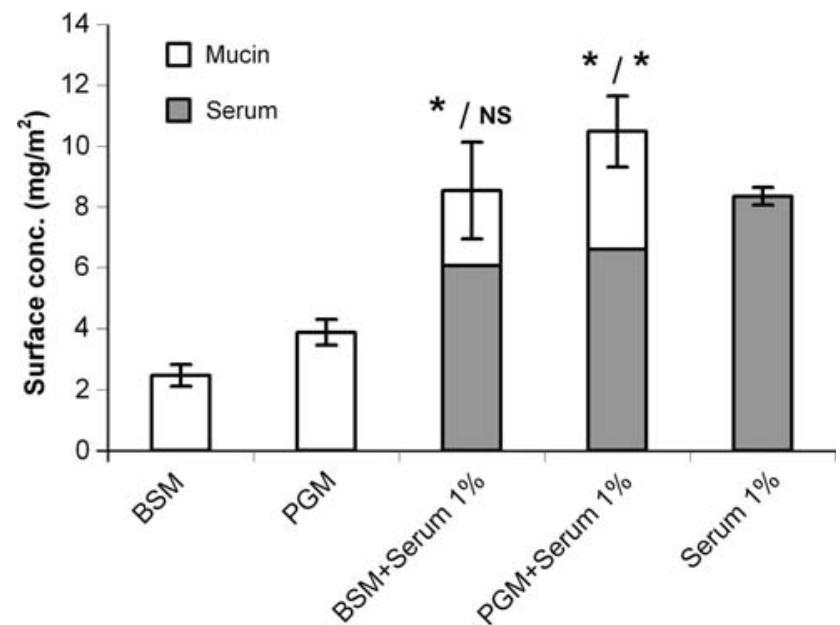

Fig. 7 Total and specific surface concentrations of mucin and/or human serum proteins on Thermanox substrates as a function of coating condition as described in Table 1. Composite layers were analyzed by combining the mBCA assay with mucin- and whole serum sensitive assays according to Sect. 2.4. Significant differences in total protein surface concentration compared to respective regular mucin coating and the serum-coated control are marked with asterisks (left for regular mucin coating and right for serum control), using the following significance levels: $* P<0.05$ and $\mathrm{NS}=$ not significant. Error bars represent mean $\pm 1 \mathrm{SD}$ for the total surface concentration. $(n=3)$

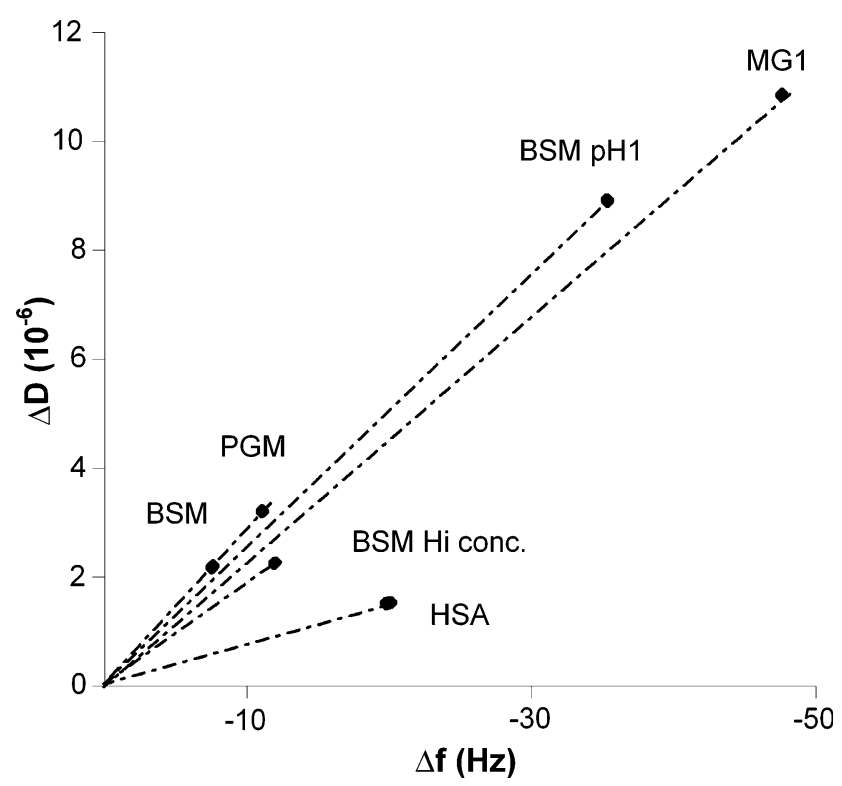

Fig. 8 QCM-D evaluation of mucin coating properties. Diagram showing the final shifts in dissipated energy $(\Delta D)$ recorded for given final shifts in frequency $(\Delta f)$ for different mucin coatings on a polystyrene model surface. All mucin coatings were formulated according to Table 1 and studied in their final relaxed states after three rinsings with PBS20. Line constructs indicate the $\Delta D / \Delta f$ slope associated with each coating. Human serum albumin (HSA) adsorbed at $0.125 \mathrm{mg} / \mathrm{ml}$ for $20 \mathrm{~h}$ served as a reference

adlayer conformations. That is, while rigid and compact layers yield low $K_{D-f}$ values, bulky and diffuse layers yield high $K_{D-f}$ values. 
Table 2 QCM-D evaluation of mucin coating properties

\begin{tabular}{|c|c|c|c|c|c|c|}
\hline Coating & $\rho_{f}\left(\mathrm{~kg} / \mathrm{m}^{3}\right)$ & $d_{f}(\mathrm{~nm})$ & $\eta_{f}(\mathrm{mPas})$ & $\mu_{f} \partial(\mathrm{kPa})$ & $\tau_{f}(\mathrm{~ns})$ & Water content $(\%)$ \\
\hline BSM & 1,016 & 46 & 1.0 & 1.5 & 698 & 95 \\
\hline PGM & 1,022 & 77 & 1.1 & 1.3 & 833 & 95 \\
\hline MG1 & 1,043 & 93 & 1.2 & 2.0 & 595 & 88 \\
\hline BSM Hi conc. & 1,066 & 46 & 1.0 & 2.3 & 435 & 90 \\
\hline BSM pH 1 & 1,020 & 84 & 1.2 & 1.6 & 725 & 94 \\
\hline HSA & 1,211 & 4.2 & 2.7 & 318 & 8 & 55 \\
\hline
\end{tabular}

Modeled effective coating density $\left(\rho_{f}\right)$, thickness $\left(d_{f}\right)$, shear viscosity $\left(\eta_{f}\right)$ and shear elastic modulus $\left(\mu_{f}\right)$ together with coating relaxation time $\left(\tau_{f}\right)$ and water content. All coatings were prepared on polystyrene model surfaces. Mucin coatings were formulated according to Table 1. Human serum albumin (HSA) adsorbed at $0.125 \mathrm{mg} / \mathrm{ml}$ for $20 \mathrm{~h}$ served as a reference

Data is presented for the BSM, PGM and MG1 mucins adsorbed under regular conditions as well as for the BSM mucin adsorbed at higher mucin concentration $(1 \mathrm{mg} / \mathrm{ml})$ and at $\mathrm{pH} 1$ (after 1 min of pre-conditioning in PBS20$\mathrm{pH} 1$ buffer). The adsorption of human serum albumin (HSA) at $0.125 \mathrm{mg} / \mathrm{ml}$ served as a reference. All coatings were formulated according to Table 1 and studied in their final relaxed states after three rinsings with PBS20. From studying Fig. 8 we find that the $K_{D-f}$ values associated with the mucin coatings arrange in the following way: $K_{D-f}(\mathrm{BSM})=K_{D-f}(\mathrm{PGM})>K_{D-f}(\mathrm{BSM} \mathrm{pH} 1)>K_{D-f}$ (MG1) $>K_{D-f}$ (BSM Hi conc.). This means that the BSM and PGM coatings formed under regular condition were the most diffusely structured coatings and that the BSM coating formed at high mucin concentration was the most compact coating. While the BSM coatings formed under regular condition and at low $\mathrm{pH}$ had similar viscoelastic properties, the amount of adsorbed mass was considerably higher for the coating formed at low $\mathrm{pH}$. A similar, high mass uptake was recorded for the MG1 coating, which had a more compact conformation than the other regular coatings, as judged from its lower $\Delta D / \Delta f$ slope.

More detailed information about the coatings was gained from fitting the QCM data to a viscoelastic model. Table 2 presents the best-fit parameters associated with each coating. Accordingly, the regular BSM and PGM coatings were found to adopt relatively diffuse conformations with low layer mobility (high relaxation times) and high water contents. In comparison to these coatings, the MG1 coating was slightly denser with lower water content and higher layer mobility. The regular BSM, PGM and MG1 coatings had effective layer thicknesses of 46,77 and $93 \mathrm{~nm}$, respectively. The BSM coating formed at higher concentration was denser and more mobile with lower water content than its corresponding regular coating. Except for a significantly higher layer thickness, the BSM coating formulated at $\mathrm{pH} 1$ showed almost identical viscoelastic properties as the regular BSM coating.

\section{Discussion}

In the present study, we quantified the production of reactive oxygen species (ROS) following neutrophil contact with differently mucin-coated substrates of a polyethylene terephthalate-based model biomaterial (Thermanox). In good agreement with our recent findings [16], the BSM, PGM and MG1 coatings were shown capable of effectively reducing the neutrophil activation normally following contact with the material surface (Fig. 2). In addition, mucin adsorption at low $\mathrm{pH}$ was proven a useful route for the production of highly surfacepassivating mucin coatings.

Although the MG1-coated substrate was associated with a seemingly high ROS level the absolute neutrophil activation induced by this substrate should be regarded very low. This is based on recent microscopic data [16], which showed the MG1-coated surface to be populated with very few neutrophils, of which all had a non-activated morphology. Such discrepancy is explained by non samplespecific neutrophil activation caused by non-specific interactions of neutrophils with the non-treated supporting plastic material. We estimate the "background signal" to be approximately $30 \%$ of the ROS signal for the noncoated and highly activating control.

Except for the PGM-I mucin, which likely was influenced by aggregation effects [27-30], the relative mucin uptakes on the Thermanox substrate (Fig. 6a) correlated well with recently found uptakes on a hydrophobic model surface [9]. In similarity with that study, we note the high mass uptake of the MG1 mucin and the concentrationdependent mass uptakes of the BSM and PGM mucins. For the coated substrates, no clear trend was found between the amount of adsorbed mucin and the degree of induced neutrophil activation. Specifically, substrates coated with mucins at high ionic strength showed significantly higher neutrophil activation levels than their corresponding substrates coated at high mucin concentration and at $\mathrm{pH} 1$ (Figs. 2, 4), despite the fact that all these substrates were coated with comparable amounts of mucin (Fig. 6b). From 
this, we concluded that the amount of adsorbed mucin is no decisive marker of mucin coating performance.

Although performed with a different model substrate, the QCM-D analysis (Fig. 8; Table 2) provided valuable structural information about the mucin coatings. Firstly, we note that the modeled thicknesses of the BSM $(46 \mathrm{~nm})$ and MG1 (93 nm) surface layers (Table 2) were in good agreement with the recently determined [9] root-meansquare radii $\left(\mathrm{R}_{\mathrm{rms}}\right)$ of the adsorbing mucin species (46 and $86 \mathrm{~nm}$, respectively). In contrast, the PGM mucin formed a considerably thicker surface layer $(77 \mathrm{~nm})$ than its $R_{\mathrm{rms}}$ value $(47 \mathrm{~nm})$ predicted. This difference was likely due to PGM multilayer formation, caused by more pronounced PGM self-association [27-30].

The QCM-D analysis helped link mucin coating performance to mucin surface conformation. Specifically, detailed data analysis showed thick expanded ("BSM pH 1") and thin compact ("BSM Hi conc.") mucin coatings to be equally surface-passivating. Although the biological significance is unclear, it is tempting to discuss these structurally different layer conformations in terms of the two main layers of the native mucosa. That is, while the outer secreted layer of the mucosa is thick with a complex, expanded conformation [31], the inner cell-bound layer adopts a more ordered, relatively thin conformation [32]. Since the electrostatic repulsion between the adsorbed mucins and the neutrophils should be largely screened at the high ionic strength used $(150 \mathrm{mM})$, we suggest surfaceshielding through steric hindrance to be the most critical factor determining mucin coating performance. This finding is well in line with previous observations [14, 15].

In the given context, the relatively thick and dense conformation of the MG1 coating, well explained its excellent performance. Possibly, the compact solution conformation and complex composition of the MG1 mucin [9] favored the formation of biocompatible coatings. Particularly, we cannot exclude the possibility that non-mucin components, associated with the MG1 mucin, possessed immune-suppressing capabilities. The mere origin of the human mucin coating suggested this coating to benefit from species-specific factors compared to the coatings formed from the porcine and bovine mucins. Interestingly however, our results showed the best-performing BSM and PGM coatings to be equally surface-passivating as the MG1 coating, thus indicating a high degree of functional homology between the mucins.

The seemingly low serum concentration ( 1 vol.\%) used in the present study was justified from a comparative point of view since we wanted to compare the biological effect of adsorbed mucin and serum components of similar surface concentrations. The mucin-serum composite coatings were associated with lower ROS production, cell adhesion and cell spreading than the coatings formed from their constituent components (Figs. 3, 5). Plausible explanations to the improved performance of the mucin coating upon serum addition were: (a) the serum components adsorbed to non-coated regions on the mucin-coated surface, thereby reducing total neutrophil-substrate contact and/or (b) the serum components adsorbed specifically to the mucin thereby forming surface arrangements with reduced activation potential and/or (c) the serum components adsorbed selectively to the mucin thereby leaving neutrophil-activating components behind in the bulk medium. Considering the diffuse organization of the regular mucin coatings (Fig. 8), alternative a) seems likely to explain a large part of the observed effect. However, given that the reductions in ROS (Fig. 3) probably were underestimated (see above), and that the differences in serum content and total adsorbed mass between the composites and the highly activating serum control were small (Fig. 7), additional factors were suggested to influence composite coating performance. In line with alternatives (b) and (c), we therefore tentatively propose a synergistic action of the mucin and serum components on the surface, wherein the mucin component could provide favorable support for the serum components. In support of such synergistic action, recent studies on a BSMbovine serum albumin system have shown several biologically relevant parameters, such as the layer viscoelasticity [33] and the interfacial friction and layer stability (unpublished data) to be significantly changed for the composite layer compared to the layers of its constituting components. As described there [33], the addition of albumin resulted in a more rigid, presumably more compact surface layer, which could resist substantial shear.

Biomaterial strategies based on tailor-made composite coatings have been developed previously; e.g. the specific and reversible adsorption of human serum albumin to a synthetically modified dextran matrix [34]. Although the in vivo performance of that particular coating has been disputed [35], the present work together with a recent study performed on combined layers of human MUC5B mucin and lactoperoxidase [36], suggest that further attention should be given the use of mucins as biomaterial surface matrices for support of biologically relevant components. Particularly, future research will focus on the interactions of neutrophils with composite coatings of mucin and specific human host proteins commonly encountered during implantation. Furthermore, the action of the coagulation and complement systems, both known to play important roles in the inflammatory process [37, 38], will be investigated.

\section{Conclusions}

In conclusion, we showed that surface coatings of bovine (BSM), porcine (PGM) and human (MG1) mucins strongly 
could reduce the neutrophil production of reactive oxygen species (ROS) normally following contact with a polymeric model biomaterial (Thermanox). Furthermore, mucin adsorption at low $\mathrm{pH}$ was proven a useful route for the production of highly surface-passivating mucin coatings. QCM-D analysis suggested efficient mucin surfaceshielding to be critical for good mucin coating performance. Interestingly, the shielding power appeared equal for thick expanded and thin compact mucin coatings. Given that mucins from three different animal sources were able to perform equally well, our results indicated a high degree of functional homology between the mucins. Finally, combined mucin-serum coatings were associated with low neutrophil activation. Particularly, since our data suggested partly synergistic mucin-serum action, we highlight the possibility that pre-adsorbed mucins could provide favorable support for adsorbing host components.

Acknowledgements We greatly acknowledge Prof. Karin Caldwell (Department of Physical and Analytical Chemistry, Uppsala University) for introducing us to the field and providing us with valuable comments during the manuscript preparation. We thank Lena Gröndahl and Riitta Laukkanen-Mållberg (Department of Clinical Chemistry and Pharmacology, Uppsala University) for help with the neutrophil isolation. Leif Ljungh (Department of Medical Cell Biology, Uppsala University) and Dr. Jonas Andersson (Department of Clinical Immunology, Uppsala University) are acknowledged for help with the SEM analysis and the human serum preparation, respectively. Finally, Prof. Per Venge (Department of Clinical Chemistry and Pharmacology, Uppsala University) is acknowledged with gratitude valuable discussions regarding the neutrophil biology.

\section{References}

1. J.M. Anderson, in Biomaterials Science, ed. by B.D. Ratner, A.S. Hoffman, F.J. Schoen, J.E. Lemons (Elsevier, Oxford, 2004), p. 296

2. A.S. Hoffman, J.A. Hubbell, in Biomaterials Science, ed. by B.D. Ratner, A.S. Hoffman, F.J. Schoen, J.E. Lemons (Elsevier, Oxford, 2004), p. 225

3. J. Perez-Vilar, in Encyclopedia of Biological Chemistry, ed. by W.J. Lennarz, M.D. Lane (Elsevier, Oxford, 2004), p. 758

4. B.J.W. Klinken, J. Dekker, H.A. Büller, A.W.C. Einerhand, Am. J. Physiol. 269, G613 (1995)

5. R. Bansil, B.S. Turner, Curr. Opin. Colloid Interface Sci. 11, 164 (2006). doi:10.1016/j.cocis.2005.11.001

6. M.A. Hollingsworth, B.J. Swanson, Nat. Rev. Cancer 4, 45 (2004). doi:10.1038/nrc1251

7. L. Shi, Trends Glycosci. Glycotechnol. 12, 229 (2000)

8. L. Shi, R. Ardehali, K.D. Caldwell, P. Valint, Colloids Surf. B 17, 229 (2000). doi:10.1016/S0927-7765(99)00121-6

9. T. Sandberg, H. Blom, K.D. Caldwell, J. Biomed. Mater. Res. A (in press). doi:10.1002/jbm.a.32266

10. R.N. Mitchell, in Biomaterials Science, ed. by B.D. Ratner, A.S. Hoffman, F.J. Schoen, J.E. Lemons (Elsevier, Oxford, 2004), p. 304

11. H. Nygren, M. Broberg, C. Eriksson, H. Sahlin, N. Yahyapour, Colloids Surf. B 22, 87 (2001). doi:10.1016/S0927-7765(00) 00216-2
12. A. Prakobphol, K. Tangemann, S.D. Rosen, C.I. Hoover, H. Leffler, S.J. Fisher, Biochemistry 38, 6817 (1999). doi: 10.1021/bi990145m

13. B.M. Fischer, J.A. Voynow, Am. J. Respir. Cell Mol. Biol. 26, 447 (2002)

14. K.P. O’Boyle, T. Chen, S. Kozlowski, Scand. J. Immunol. 52, 46 (2000). doi:10.1046/j.1365-3083.2000.00756.x

15. M.L.R. Aknin, M. Berry, A.D. Dick, D. Khan-Lim, Cell Tissue Res. 318, 545 (2004). doi:10.1007/s00441-004-0957-8

16. T. Sandberg, J. Carlsson, M. Karlsson Ott, Microsc. Res. Tech. 70, 864 (2007). doi:10.1002/jemt.20489

17. S.L. Cooper, S.A. Visser, R.W. Hergenrother, N.M.K Lamba, in Biomaterials Science, ed. by B.D. Ratner, A.S. Hoffman, F.J. Schoen, J.E. Lemons (Elsevier, Oxford, 2004), p. 67

18. P.K. Smith, R.I. Krohn, G.T. Hermanson, A.K. Mallia, F.H. Gartner, M.D. Provenzano et al., Anal. Biochem. 150, 76 (1985). doi:10.1016/0003-2697(85)90442-7

19. B.T. Doumas, Clin. Chem. 21, 1159 (1975)

20. T. Sandberg, L. Mellin, U. Gelius, K.D. Caldwell, J. Colloid Interface Sci. (Submitted)

21. L. Håkansson, P. Venge, Scand. J. Immunol. 11, 271 (1980). doi: 10.1111/j.1365-3083.1980.tb00235.x

22. C. Dahlgren, A. Karlsson, J. Immunol. Methods 232, 3 (1999). doi:10.1016/S0022-1759(99)00146-5

23. F. Höök, M. Rodahl, P. Brzezinski, B. Kasemo, Langmuir 14, 729 (1998). doi:10.1021/la970815u

24. M.V. Voinova, M. Rodahl, M. Jonsson, B. Kasemo, Phys. Scr. 59, 391 (1999). doi:10.1238/Physica.Regular.059a00391

25. J. Tsai, R. Taylor, C. Chothia, M. Gerstein, J. Mol. Biol. 290, 253 (1999). doi:10.1006/jmbi.1999.2829

26. T. Tammelin, J. Merta, L.-S. Johansson, P. Stenius, Langmuir 20, 10900 (2004). doi:10.1021/la0487693

27. X. Cao, R. Bansil, K.R. Bhaskar, B.S. Turner, J.T. Lamont, N. Niu et al., Biophys. J. 76, 1250 (1999)

28. S. Lee, M. Muller, R. Kurosch, N.D. Spencer, Langmuir 21, 8344 (2005). doi:10.1021/la050779w

29. L.E. Bromberg, D.P. Barr, Biomacromolecules 1, 325 (2000). doi:10.1021/bm005532m

30. Z. Hong, B. Chasan, R. Bansil, B.S. Turner, K.R. Bhaskar, N.H. Afdhal, Biomacromolecules 6, 3458 (2005). doi:10.1021/ bm0505843

31. D.J. Thornton, K. Rousseau, M.A. McGuckin, Annu. Rev. Physiol. 70, 459 (2008). doi:10.1146/annurev.physiol.70.113006.100702

32. C.L. Hattrup, S.J. Gendler, Annu. Rev. Physiol. 70, 431 (2008). doi:10.1146/annurev.physiol.70.113006.100659

33. A.A. Feiler, A. Sahlholm, T. Sandberg, K.D. Caldwell, J. Colloid Interface Sci. 315, 475 (2007). doi:10.1016/j.jcis.2007.07.029

34. J.R. Keogh, F.F. Velander, J.W. Eaton, J. Biomed. Mater. Res. 26, 441 (1992). doi:10.1002/jbm.820260403

35. J.M. Kao, R. Rose, M. Yousef, S.K. Hunter, V.G.J. Rodgers, J. Biomed. Mater. Res. 47, 537 (1999). doi:10.1002/(SICI)10974636(19991215)47:4<537::AID-JBM10>3.0.CO;2-I

36. L. Lindh, I.E. Svendsen, O. Svensson, M. Cardenas, T. Arnebrant, J. Colloid Interface Sci. 310, 74 (2007). doi:10.1016/ j.jcis.2007.01.086

37. S.R. Hanson, in Biomaterials Science, ed. by B.D. Ratner, A.S. Hoffman, F.J. Schoen, J.E. Lemons (Elsevier, Oxford, 2004), p. 332

38. R.J Johnson, in Biomaterials Science, ed. by B.D. Ratner, A.S. Hoffman, F.J. Schoen, J.E. Lemons (Elsevier, Oxford, 2004). p. 318 